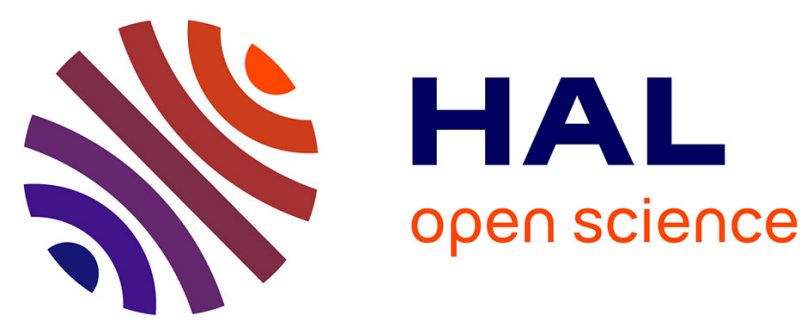

\title{
Handling soft constraints in hoist scheduling problems: the fuzzy approach
}

Hélène Fargier, Jacques Lamothe

\section{To cite this version:}

Hélène Fargier, Jacques Lamothe. Handling soft constraints in hoist scheduling problems: the fuzzy approach. Engineering Applications of Artificial Intelligence, 2001, 14 (3), p.387-399. 10.1016/S09521976(01)00008-2 . hal-01618010

\section{HAL Id: hal-01618010 https://hal.science/hal-01618010}

Submitted on 6 Nov 2018

HAL is a multi-disciplinary open access archive for the deposit and dissemination of scientific research documents, whether they are published or not. The documents may come from teaching and research institutions in France or abroad, or from public or private research centers.
L'archive ouverte pluridisciplinaire HAL, est destinée au dépôt et à la diffusion de documents scientifiques de niveau recherche, publiés ou non, émanant des établissements d'enseignement et de recherche français ou étrangers, des laboratoires publics ou privés. 


\title{
Handling soft constraints in hoist scheduling problems: the fuzzy approach
}

\author{
Hélène Fargier ${ }^{\mathrm{a}, *}$, Jacques Lamothe ${ }^{\mathrm{b}}$ \\ ${ }^{a}$ Institut de Recherche en Informatique de Toulouse, 118 route de Narbonne, 31062 Toulouse Cedex, France \\ ${ }^{\mathrm{b}}$ Ecole des Mines d'Albi-Carmaux, Campus Jarlard, Route de Teillet, 81000 Albi Cedex 09, France
}

\begin{abstract}
The Hoist Schedu ling Problem (HSP) deals with the schedu ling of hoists that move products between tanks in electroplating facilities that perform chemical surface treatments. In HSP, the gradual effect of soaking times (operation duration in tanks) on the quality of treatment can be represented by means of fuzzy sets: the satisfaction degree in a fuzzy interval models a quality evaluation of the chemical treatment. When temporal bou nds are requ ired, an implicit relaxation of these flexible constraints can thus be performed so as to meet the due-date. When the objective is rather a minimization of the makespan, a bi-criteria decision problem has to be dealt with that involves both the quality and the line throughputoptimization. Rather than an aggregation of the two evaluations under the form of a single criterion, we propose a decision-support approach that quickly converges to a good trade-off between the two criteria.
\end{abstract}

Keywords: Fuzzy logic; Hoist scheduling; Fuzzy constraints

\section{Introduction}

When batch processing times on workstations are close to batches travelling times, controlling and scheduling the movements of the batches becomes necessary for process performance. This point is crucial in chemical treatment or electroplating lines, since the chemical process strongly limits the in-process inventories and consequently the line flexibility and productivity. However, electroplating and surface treatment are widely used in manufacturing processes and, as a result, are often the cause of a bottleneck.

This situation has resulted in much research work over the last 20 years on the scheduling problem of hoists that move products between tanks in automated electroplating lines, called Hoist Scheduling Problem (HSP). In this kind of scheduling problem, the duration of the operations can be chosen in an interval. The

\footnotetext{
*Corresponding author.

E-mail addresses: fargier@irit.fr (H. Fargier), lamothe@enstimac.fr (J. Lamothe).
}

definition of the bounds of this interval results from a trade-off between two aims:

- ensuring the quality surface treatment by defining a short interval,

- generating flexibility by fixing a large interval so that line productivity can be increased.

Usually, this trade-off is defined a priori without identifying consequences on productivity. Thus, the HSP consists in controlling the hoist moves in order to maximize productivity while satisfying given bounds on workstation processing times.

In this paper we propose a fuzzy approach to the HSP that allows to handle the gradual effect of soaking times on the quality of treatment: in fuzzy HSP the duration of an operation is selected in a fuzzy interval, the satisfaction degree of which is a quality evaluation of the chemical treatment. The trade-off between quality and productivity then clearly results from a bi-criteria decision process.

Fuzzy approaches have already been adopted in PERT problems (Chanas and Kamburowski, 1981; 
Lootsma, 1989; Nasution, 1993) and more recently in project scheduling (Hapke et al., 1994; Hapke and Slowinski, 1996), in master production scheduling (Fargier and Thierry, 1999) and in job-shop or flowshop scheduling (Dubois, 1989; Kerr and Walker, 1989; Ishii et al., 1992; Ishibushi et al., 1994a, b; Dubois et al., 1995; Ptuskin, 1995; Fargier, 1996; Fortemps, 1997). Fuzzy sets usually represent uncertain time parameters like due-dates and durations, or preference levels for parameters. However, these works either consider only one optimization criterion or make the assumption that the criteria may be aggregated a priori so as to define only one final and global decision criterion. The latter approach becomes tricky when two criteria such as the quality and the makespan optimization (as is the case in HSP) are considered. To avoid having to follow this strong hypothesis, we would rather let the users make their own trade-off between both criteria: we adopt an approach oriented toward decision support.

The fuzzy approach will be analyzed in the context of the dynamic HSP control organization of the line. However, it should be noted that the fuzzy analysis can be the same for other kinds of HSP control organization. Our conclusions may lead to a reappraisal and application to classical HSP models and resolution procedures that would follow the same logic as the ones presented in this paper.

The paper is organized as follows. Section 2 presents the various HSPs and more particularly the Local Problem of dynamic HSP. The fuzzy model of this particular HSP is described in Section 3. Then, Section 4 introduces resolution procedures for the fuzzy model. Finally, in Section 5, we propose a decision support approach that may help the user converge rapidly on a trade-off between both criteria.

\section{Classical HSPs}

\subsection{Definition of the various $H S P S$}

An electroplating is a chemical treatment that consists in laying a thin and homogeneous chemical deposit of materials on a product. Generally, a chemical treatment line performs different elementary treatments. Each one requires a series of chemical reactions made in tanks. As a result, depending on the desired treatment, a product will have some of the elementary treatments in a fixed sequence.

In fact, a chemical treatment line is composed of (see Fig. 1):

- Tanks, each of them containing a chemical agent. Tanks can contain active reagents such as acids in order to strip product surfaces or an electroplating process which deposits a metal layer on the product surfaces. They can also contain inactive reagents such as water for cleaning.

- Loading and unloading workstations and buffers. At the loading workstation, products that will have the same treatment are secured on a carrier. At the unloading workstation, products are removed from the carrier.

- Hoists which move carriers from tank to tank, one by one, depending on the carrier routing. Collisions between hoists must be avoided as they move on the same track. The hoist charge is then composed of:

$\circ$ loaded hoist movements to move carriers from tank to tank, the duration of which is specified in the carrier routing;

$\circ$ empty hoist movements to go to the next starting tank or to avoid a collision with another hoist. The duration of these empty movements depends on the hoist schedule.

Therefore, the electroplating process results from the soaking of a carrier in tanks depending on the carrier routing.

Due to the chemical process, the following specific constraints must also be considered:

1. Once a carrier finishes its processing in a tank, it must go to the next one as quickly as possible. This means that there are no in-process buffers.

2. The soaking time of a given carrier in a tank (i.e. the time a given carrier needs to complete a chemical reaction in a tank) is not given precisely. But in

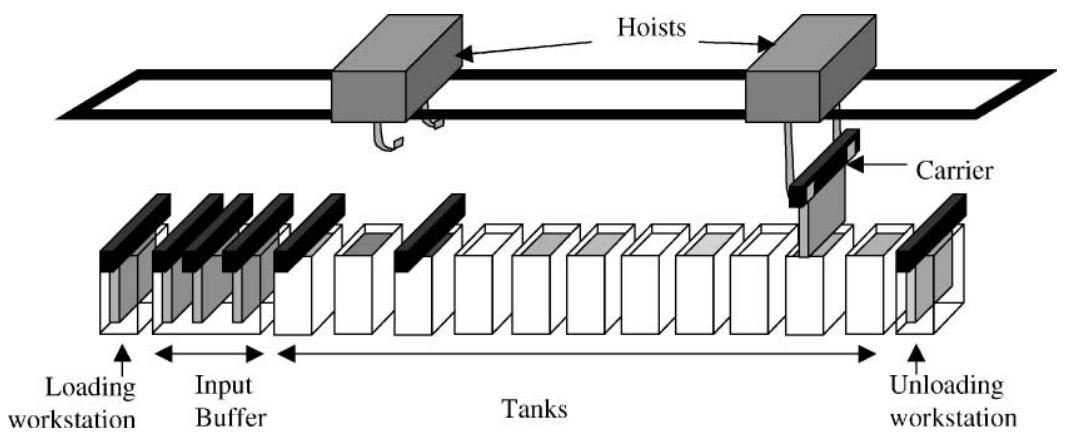

Fig. 1. A chemical treatment line. 
classical HSP, a minimum and a maximum soaking time limit is supposed to be pre-established in the carrier routing.

These specific constraints over-constrain the scheduling problem of the line. They also imply that one has to make sure that a carrier can go through the whole line once it enters the line. So each of the hoists movements has to be forecast before a carrier enters the line. The control of the hoist with respect to all these constraints is known as the Hoist Scheduling Problem (HSP).

The previous definition of HSP is rather a physical description of the line. Depending on the control organization of the line, four different formulations of HSP have been distinguished, namely CHSP, RHSP, PHSP and DHSP. A detailed typology and notation of HSPs can be found in Bloch and Manier (1999).

The Cyclic Hoist Scheduling Problem (CHSP) consists in finding a cycle of hoist movements that will be repeated indefinitely. Such research works suppose that all carrier routings are identical. But then, an optimal productivity cyclic production is generally found using a branch and bound algorithm (see, for instance, Phillips and Unger, 1976; Shapiro and Nuttle, 1988; Armstrong et al., 1994; Lacoste and Baptiste, 1992; Lei and Wang, 1994; Hanen, 1994; Ng, 1996; Chen et al., 1998). Interestingly, fuzzy processing times are introduced in Ptuskin (1995) so as to model the quality level of the operations, but no trade-off is proposed between the productivity criterion and the quality: the difficulty is ruled out through a de-fuzzification of the problem, namely the choice of a level of quality, before the search of a cyclic schedule is carried out.

In the Real-time Hoist Scheduling Problem (RHSP), carriers can have different routings but the maximal soaking time limit can be moderately exceeded. Thus, each carrier movement no longer needs to be forecast because a carrier can remain within a tank as long as necessary. In this context, the problem consists in determining in real time what will be the next movement of the hoist. Then, rule-based systems (Yih, 1990; Sun et al., 1994; Song et al., 1993) or neural network (Min et al., 1998) lead to a trade-off between the line throughput rate and the violation of the limit soaking time. It is important to note that the trade-off between productivity and quality cannot be controlled once the rules or the neural network have been defined. It can only be evaluated afterwards.

The Predictive Hoist Scheduling Problem (PHSP) considers that a list of jobs with different routings is given. The aim consists in determining the sequence of jobs that get into the line and also the schedule of the hoist movements in order to minimize the makespan. In this context, Fleury et al. (1996) introduce a multi-agent procedure while Caux et al. (1992, 1995) and Norre
(1999) describe the cooperation between a simulated annealing procedure (that computes the sequence of entering jobs) and a simulation procedure (that simulates a rule-based RHSP).

The fourth formulation is the Dynamic Hoist Scheduling Problem (DHSP) in which jobs can be different and arrive at the loading workstation randomly. In fact, it is considered that job arrival cannot be forecast with enough precision to allow the use of a PHSP approach. Consequently, when a new job arrives (randomly), given the state of the line (in process and in buffer jobs) a new schedule of all the jobs is computed solving the so-called Local Problem (see Fig. 2). The global objective of the DHSP then consists in maximizing the line throughput after a series of Local Problems. But experience has shown that optimizing makespan (or looking for small makespan values) in each Local Problem is a good strategy to approach the global objective (Lamothe et al., 1995).

In general, a heuristic algorithm is used in order to solve a Local Problem. This procedure assumes that the sequence of the jobs already in process does not change and just tries to compute a feasible solution rapidly (Yin and Yih, 1992; Yih, 1994). The line throughput has been improved by a greedy algorithm which does not make any assumption (Ge and Yih, 1995). A branch cutting heuristic in a branch-and-bound procedure has also increased productivity (Lamothe et al., 1995). From another point of view, simulated annealing and genetic procedures that optimize makespan in Local Problems have been studied (Lim, 1997; Bloch et al., 1996).

\subsection{DHSP Local Problem formulation}

The model below refers to a classical formulation of a DHSP Local Problem. The line is supposed to have one hoist only, and any tank capacity equals one. This model can easily be generalized to a multi-hoist line without any change in the model structure: only the parameter $H_{(i, j) /(k, l)}$ would change (see for details Lamothe et al., 1996).

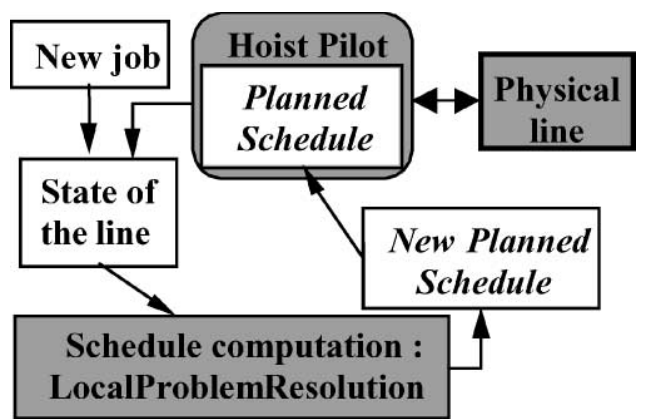

Fig. 2. Principle of the dynamic HSP. 
When a new job (carrier) arrives at the loading workstation at time $t_{0}$, the Hoist Pilot (see Fig. 2) gives the state of the in-process jobs. Then, the Local Problem consists in finding a new schedule for any in-process or in-buffers jobs. The following notations are described in Fig. 3:

- $N$ is the number of jobs.

- $t_{0}$ the arrival time of the new job.

- $O_{i, j}$ refers to the operation $j$ of job $i\left(0 \leqslant j<n b_{-} o p(i)\right)$. As there are no in-process buffers, $O_{i, j}$ refers to both the soaking operation in a tank and the loaded hoist move that brings the carrier into this tank. By convention, the last operation of each job is its uploading, which can be identified as a soaking operation with duration zero.

- init $(i)$ is the rank number of the last in-process operation of job $i$ at time $t_{0}\left(0 \leqslant \operatorname{init}(i)<n b_{-} o p(i)\right)$.

- $b_{i, j}$ is the tank position for the soaking of operation $O_{i, j}$.

- $t_{i, j}\left(1 \leqslant i \leqslant N, 1 \leqslant\right.$ init $\left.(i) \leqslant j \leqslant n b_{-} o p(i)\right)$ is the starting time of operation $O_{i, j}$ soaking in tank $b_{i, j}$. It is also the ending time of the loaded move $O_{i, j-1}$ to tank $b_{i, j}$.

- $s_{i, j}$ refers to the soaking time of operation $O_{i, j}$ in tank $b_{i, j}$.

- $S_{i, j}$ is the admissible interval for the soaking time of operation $O_{i, j}: S_{i, j}=\left[m_{i, j}, M_{i, j}\right]$.

- $c_{i, j}$ is the loaded hoist travel time when moving job $i$ from operation $O_{i, j-1}$ to operation $O_{i, j}$, thus from position $b_{i, j-1}$ to position $b_{i, j}$.

- $d_{u, v}$ is the empty hoist travel time from position $u$ to position $v$.

- $H_{(i, j) /(k, l)}$ is the minimum delay between $t_{i, j}$ and $t_{k, l}$, if the hoist movement of operation $O_{i, j}$ is done just before the hoist movement of operation $O_{k, l}$. It takes into account an empty hoist movement from position $b_{i, j}$ to position $b_{k, l-1}$ plus the loaded hoist travel from position $b_{k, l-1}$ to position $b_{k, l}$ (see Fig. 3). Thus $H_{(i, j) /(k, l)}=d_{b_{i, j}, b_{k, l-1}}+c_{k, l}$.

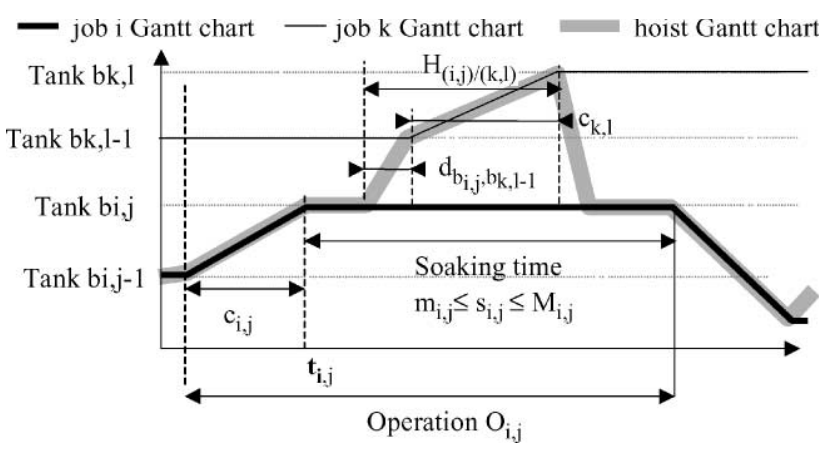

Fig. 3. Description of the notations.
In the following, we propose a formulation of the DHSP Local Problem by means of linear inequations. In order to represent the disjunctive constraints of the problem, we need binary decision variables $x_{(i, j) /(k, l)}$ :

- $x_{(i, j) /(k, l)}$ is a binary decision variable:

$\circ x_{(i, j) /(k, l)}=0$ if the loaded movement of operation $O_{i, j}$ is made before the loaded hoist movement of operation $O_{k, l}$.

$\circ$ Otherwise $x_{(i, j) /(k, l)}=1$.

The constraints are expressed below. Note that

$i \in[0 ; N-1], j \in\left[\operatorname{init}(i) ; n b_{-} o p(i)-1\right]$,

$k \in[0 ; N-1], l \in\left[\operatorname{init}(k) ; n b_{-} o p(k)-1\right]$.

- Logical constraint on the binary variables:

$$
\begin{gathered}
x_{(i, j) /(k, l)} \in\{0 ; 1\} ; \\
\forall(i, j, k, l) \text { s.t. }(i, j) \neq(k, l): \\
x_{(i, j) /(k, l)}+x_{(k, l) /(i, j)}=1
\end{gathered}
$$

(a sequence of loaded moves of the hoist is looked for: either $O_{i, j}$ is made before $O_{k, l}$, or $O_{k, l}$ is made before $O_{i, j}$ )

$$
\begin{aligned}
& x_{(i, j) /(i, j)}=1, \\
& x_{(i, j) /(i, j+1)}=0 \quad\left(O_{i, j} \text { is before } O_{i, j+1}\right), \\
& x_{(i, j) /(k, l)} \leqslant x_{(i, j+1) /(k, l)} \\
& \text { (if } \left.O_{i, j+1} \text { precedes } O_{k, l}, \text { then so does } O_{i, j}\right) .
\end{aligned}
$$

- Initialization constraints due to the state of the line at time $t_{0}$ :

$$
\left\{\begin{array}{c}
\text { if job } i \text { is already in process at } \\
t_{0}: t_{i, \text { init }(i)}-t_{0} \text { is given, } \\
\text { otherwise, } t_{i, \text { init }(i)}-t_{0} \geqslant 0 .
\end{array}\right.
$$

- The capacity of the hoist is 1, i.e. it cannot move two different jobs simultaneously. Moreover, the shortest time between two operations is obtained when they are performed in succession. We thus get the following disjunctive constraint for any pair of operations $O_{i, j}$ and $O_{k, l}$ :

$t_{k, l}-t_{i, j} \geqslant c_{k, l}+d_{b_{i, j}, b_{k, l-1}}$

or

$t_{i, j}-t_{k, l} \geqslant c_{i, j}+d_{b_{i, j}, b_{k, l-1}}$.

This can also be expressed as $t_{k, l}-t_{i, j} \geqslant H_{(i, j) /(k, l)}$ or $t_{i, j}-t_{k, l} \geqslant H_{(k, l) /(i, j)}$.

Using the binary decision variables $x_{(i, j) /(k, l)}$, this constraint is now expressed as two linear 
inequalities:

$$
\begin{aligned}
& t_{k, l}-t_{i, j} \geqslant H_{(i, j) /(k, l)}-x_{(i, j) /(k, l)} M, \\
& t_{i, j}-t_{k, l} \geqslant H_{(k, l) /(i, j)}-x_{(k, l) /(i, j)} M,
\end{aligned}
$$

where $M$ is a very high fixed number.

- Capacity of the tanks: A tank cannot carry out two jobs simultaneously. Considering two operations $O_{k, l}$ and $O_{i, j}$ using the same tank $T\left(b_{i, j}=b_{k, l}=T\right)$ :

- If operation $O_{k, l}$ follows operation $O_{i, j}$ into the tank $\left(x_{(i, j) /(k, l)}=0\right)$, carrier $i$ must first have been moved to its next location $\left(b_{i, j+1}\right)$ by the hoist before carrier $k$ reaches tank $T\left(x_{(i, j+1) /(k, l)}=0\right)$. Thus, hoist movements will respect the following sequence: $O_{i, j}, O_{i, j+1}, O_{k, l}, O_{k, l+1}$. So we get

$$
\begin{gathered}
x_{(i, j) /(k, l)}=x_{(i, j+1) /(k, l)}=x_{(i, j) /(k, l+1)} \\
=x_{(i, j+1) /(k, l+1)}=0 .
\end{gathered}
$$

- If operation $O_{k, l}$ precedes operation $O_{i, j}$ into the tank $\left(x_{(i, j) /(k, l)}=1\right)$, carrier $k$ must first have been moved to its next location $\left(b_{k, l+1}\right)$ by the hoist before carrier $i$ reaches tank $T$ $\left(x_{(i, j) /(k, l+1)}=1\right)$. Thus hoist movements will respect the following sequence: $O_{k, l}, O_{k, l+1}, O_{i, j}$, $O_{i, j+1}$. So we get

$$
\begin{gathered}
x_{(i, j) /(k, l)}=x_{(i, j+1) /(k, l)}=x_{(i, j) /(k, l+1)} \\
=x_{(i, j+1) /(k, l+1)}=1 .
\end{gathered}
$$

Hence, for any pair of operations $O_{i, j}$ and $O_{k, l}$ such as $b_{i, j}=b_{k, l}$ we get

$$
x_{(i, j) /(k, l)}=x_{(i, j+1) /(k, l)}=x_{(i, j) /(k, l+1)}=x_{(i, j+1) /(k, l+1)} \text {. }
$$

- Chemical specific constraints: As there cannot be any in-process inventories, the soaking time of operation $O_{i, j}$ in the tank $b_{i, j}$ is $s_{i, j}=t_{i, j+1}-t_{i, j}-c_{i, j+1}$. This soaking time is bounded: $s_{i, j} \in S_{i, j=[} m_{i, j}, M_{i, j]}$, We thus get the inequalities

$$
m_{i, j} \leqslant t_{i, j+1}-t_{i, j}-c_{i, j+1} \leqslant M_{i, j} .
$$

According to this formulation of the constraints, an admissible solution of a HSP problem is an assignment of the decision variables $t_{i, j}$ and $x_{(i, j) /(k, l)}$ that satisfies the linear inequalities (1)-(5). When the makespan is considered as discrimination criterion, the optimal solutions of the problem are the admissible solutions that minimize the quantity

$$
\max _{i} t_{i, n b_{-} o p(i)-1}
$$

since the last operation of each job is by convention its unloading.
In some situations, obtaining a good makespan rather than an optimal one is considered as a reasonable approach. The idea is thus to introduce the completion constraint

$\forall i, t_{i, n b_{-} o p(i)-1} \leqslant B$.

When different completion times are required for different jobs, these temporal constraints can be expressed as

$t_{i, n b_{-} o p(i)-1} \leqslant B_{i}$,

where $B_{i}$ is the completion time of job $i$.

The inequations (1)-(5), and (6b) define a HSP with temporal bounds.

\section{Fuzzy HSP}

\subsection{Fuzzy model of preferences on soaking times}

In classical HSP, it is considered that the chemist defining the line gives the process characteristics in order to ensure the quality of any job treated on this line. As a result, he defines the possible routings, the minimum and maximum bounds on soaking times, the in-tankreagent life-cycle. This approach is not fully satisfactory for the following reasons:

- Usually, the chemist defines a reference duration, and admissible deviations from it, for each tank and each routing. These admissible deviations may be negotiated in order to enable line throughput optimization. This means that all durations are not equivalent within the admissible interval $\left[m_{i, j}, M_{i, j}\right]$. Some are better because they ensure a higher efficiency of the reaction and the stability of quality whatever unpredictable deviations of the reaction (reagent pollution, etc.) may occur. Such preferred durations usually do not correspond to end values of the admissible intervals which, by definition, depict the limits of the efficiency of the reaction.

- The chemist usually has tolerances on soaking durations in order to avoid risks. The minimal value is often overestimated because it must be reliable. A gradation of the impact of soaking durations on the treatment of the quality should help controlling these margins.

- The more often a tank is used, the less efficient the reagent is. As a result, the reference duration must be increased as the reagent becomes older.

- Short breakdowns may occur on the hoist (e.g. when the hoist jams above a tank, it does not detect a tank, it does not stop above the desired tank, etc.) or on tanks (when heating, electrolysis, mixing systems break down). Then, it can become impossible to find a schedule that verifies all the chemical constraints (5) of all the in-process carriers. Then, one would like to 


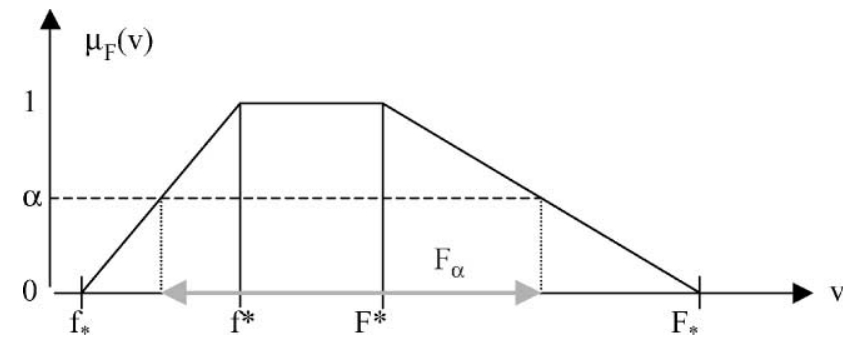

Fig. 4. A fuzzy set $F$ and its cut of level $\alpha$.

find a schedule that optimizes the treatment quality of the carriers.

Hence, it appears that the duration of the operation is subject to gradual preferences: a model that encompasses the gradual effect of soaking times on the quality of treatment is thus necessary. Following Ptuskin (1995) we propose the definition of a fuzzy domain for the soaking time of each operation.

Remember ${ }^{1}$ that a fuzzy subset $F$ of a referential $\Omega$ is defined by a membership function $\mu_{F}(v)$ that associates a membership degree in $[0,1]$ to each value $v$ in $\Omega$ : the values that are perfectly part of $F$ receive the degree 1 ; the values that do not belong to $F$ receive the degree 0 . In between, the membership is a matter of degree: the higher $\mu_{F}(v)$, the more $v$ belongs to $F$. Any value that receives a positive degree of membership belongs to the support of $F$, which is the classical set $\{v \in \Omega$, such that $\left.\mu_{F}(v)>0\right\}$. The values that perfectly belong to $F$ belong to the core of $F$, which is the classical set $\{v \in \Omega$, such that $\left.\mu_{F}(v)=1\right\}$. More generally, the $\alpha$-cut of a fuzzy set $F$ is the classical set $F_{\alpha}=\left\{v, \mu_{F}(v) \geqslant \alpha\right\}$ (Fig. 4).

Here, the fuzzy domain $S_{i, j}$ is interpreted as follows: the values of the soaking time that are not admissible receive the membership degree 0 . The support of $S_{i, j}$ represents the set of all admissible values and its core is the optimal range of soaking time for operation $O_{i, j}$. More generally, $\mu_{S_{i, j}}(v)>\mu_{S_{i, j}}(u)$, which means that the soaking duration $v$ is preferred to the duration $u$ for operation $O_{i, j}$.

The shape of the fuzzy domain $S_{i, j}$ represents how the chosen value will impact on the quality of the chemical reaction of an operation. $S_{i, j}$ is obviously a fuzzy interval, i.e. a convex fuzzy set: all its $\alpha$-cuts are classical intervals. As shown in Figs. 5 and 6, $\left[m_{i, j}^{*}, M_{i, j}^{*}\right]$ denote its cut of level 1 (i.e. its core): $m_{i, j}^{*}$ (resp. $M_{i, j}^{*}$ ) is the lowest (resp. the highest) of the ideal values for the soaking time of operation $O_{i, j}$. In the same way, the lowest (resp. the highest) of the admissible values will be denoted by $m_{i, j_{*}}\left(\right.$ resp. $M_{i, j_{*}}$ ). Now, since the shape of $S_{i, j}$ depends on various factors such as the kinetics of the chemical reaction, the degradation of the reagents,

\footnotetext{
${ }^{1}$ For more details about fuzzy sets, the reader should refer to Dubois et al. (1988)
}

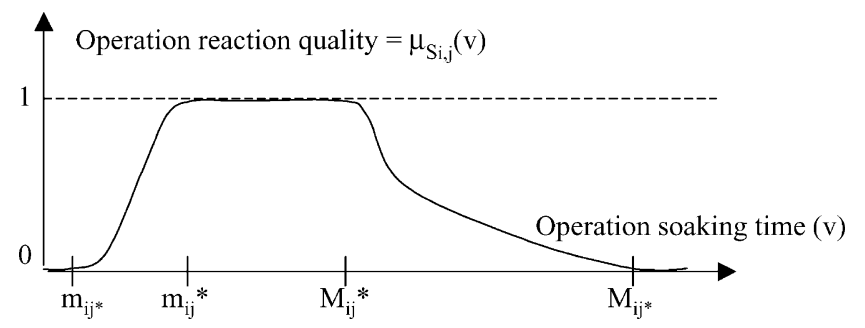

Fig. 5. Fuzzy model of an operation soaking time domain.

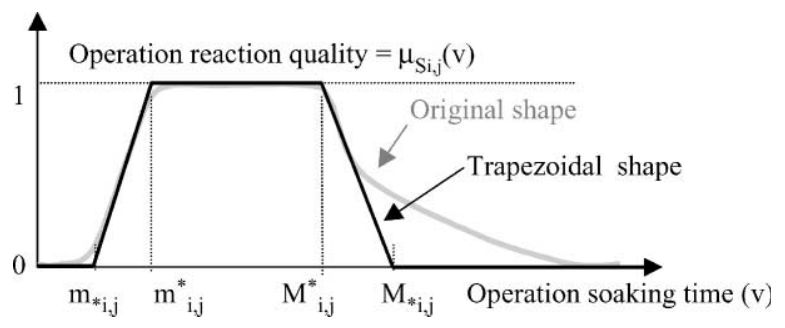

Fig. 6. Trapezoidal fuzzy model of an operation soaking time domain.

etc., it is not necessarily triangular or trapezoidal. However, shapes like the one depicted in Fig. 5 are not presently accessible for each soaking operation of the routings. On the contrary, a trapezoidal approximation of the function (see Fig. 6) is accessible in practice and will enable more efficient computations to be performed. Indeed, it can be easily encoded by the 4-uplet of parameters $\left(m_{i, j_{*}}, m_{i, j}^{*}, M_{i, j}^{*}, M_{i, j_{*}}\right)$-see Section 5 .

\subsection{Satisfaction degree of a fuzzy HSP}

Introducing preferences on the soaking time obviously affects the formulation of the DHSP Local Problem, namely the handling of the flexible chemical constraints. As in a classical HSP, a solution is an assignment $A$ of the decision variables $t_{i, j}$ and $x_{(i, j) /(k, l)}$. To be admissible, $A$ must still satisfy the logical constraints (1), the initialization constraints (2), and the capacity constraints (3), (4) that remain crisp. But the satisfaction of the chemical constraints (5) is now a matter of degree: each of them is now satisfied to a given degree $\mu_{S_{i, j}}\left(t_{i, j+1}-t_{i, j}-c_{i, j+1}\right)$. In order to evaluate an assignment $A$, we need to define its global satisfaction degree, $\operatorname{Sat}(A)$, from these local satisfaction degrees.

Given an assignment, its satisfaction degree is the aggregation of the satisfaction degrees of the carriers which result from the aggregation of the satisfaction degrees of their operations. In our model, the satisfaction degree evaluates the quality of the treatment. And, in terms of quality, the treatment of a carrier is considered as non-satisfactory as soon as one soaking operation has been badly performed: an incomplete reaction into a tank cannot be counterbalanced by a 
longer reaction in another tank. As a consequence, a carrier satisfaction degree should be evaluated as the lowest soaking operation satisfaction degree (this can be set off with a cost evaluation that would result in a sum of satisfaction degrees). In the same way, the global satisfaction degree of a fuzzy HSP assignment is the lowest carrier satisfaction degree. More formally, the satisfaction level of an assignment $A$ is defined as

$\operatorname{Sat}(A)$

$$
=\left\{\begin{array}{l}
0 \text { if a crisp constraint (1)-(4) is violated, } \\
\min \operatorname{Min}_{o_{i, j}} \mu_{S_{i, j}}\left(t_{i, j+1}-t_{i, j}-c_{i, j+1}\right) \text { otherwise. }
\end{array}\right.
$$

In other words, an assignment satisfying all crisp constraints satisfies the fuzzy scheduling problem insofar as it satisfies the least satisfied flexible constraint. The assignments violating the admissible range of soaking time for an operation $O_{i, j}$ (i.e. such that $t_{i, j+1}-$ $t_{i, j}-c_{i, j+1}>M_{i, j}^{*}$ or $\left.t_{i, j+1}-t_{i, j}-c_{i, j+1<m_{i, j *}}\right)$, are not admissible $(\operatorname{Sat}(A)=0)$, whereas the ones that satisfy all the optimal soaking windows are the best ones $(\operatorname{Sat}(A)=1)$. In between, the assignments more or less satisfy the preferences expressed on the soaking times: the less important the relaxation of the optimal soaking bounds is, the better the solution. ${ }^{2}$ Sat induces a total ordering over the solutions of the problem defined by the capacity, logical and initialization constraints. This degree can be understood as a degree of membership of the assignments to the fuzzy set of more or less admissible solutions of the FHSP.

\section{Fuzzy HSP with temporal constraints}

When temporal bounds exist, temporal constraints (6b) are required in addition to constraints (1)-(4); the admissibility Sat degree of the fuzzy HSP thus becomes

$$
\begin{aligned}
& \operatorname{Sat}(A) \\
& \quad=\left\{\begin{array}{l}
0 \text { if a crisp constraint }(1)-(4),(6 \mathrm{~b}) \text { is violated, } \\
\min _{O_{i, j}} \mu_{S_{i, j}}\left(t_{i, j+1}-t_{i, j}-c_{i, j+1}\right) \text { otherwise. }
\end{array}\right.
\end{aligned}
$$

Notice that it may happen that no sequence can perfectly satisfy all the constraints that are partially conflicting: no sequence supports the satisfaction degree 1. The satisfaction degree of the best solution, i.e. the

\footnotetext{
${ }^{2}$ In the trapezoidal model, it should be noticed that the degrees of satisfaction of the flexible constraints can be interpreted in terms of safety ranges: $\mu_{S_{i, j}}\left(t_{i, j+1}-t_{i, j}-c_{i, j+1}\right)$ is the fraction of the ranges $m_{i, j}^{*}-m_{i, j_{*}}\left(\operatorname{resp} . M_{i, j_{*}}-M_{i, j}^{*}\right)$ which is left between the soaking time of the operation and its minimal (resp. maximal) acceptable soaking time. The present approach thus looks for a temporally safe schedule: $\operatorname{Sat}(A)$ represents the minimal fraction of the flexibility ranges which are left between the soaking time of the operations and their acceptable soaking time.
}

degree Cons $=\max _{\left(A_{1}, \ldots, A_{m}\right)} \operatorname{Sat}\left(A_{1}, \ldots, A_{m}\right)$, indicates to what extent there exists a solution which satisfies all the constraints. It can be understood as the feasibility degree of the problem: a degree equal to 1 means that there is no need to relax chemical constraints to satisfy the temporal bounds. A Cons degree equal to 0 expresses a total conflict between the data of the problem: even a relaxation of the flexible constraints up to the extreme acceptable soaking time does not provide a solution that satisfies the completion times. When the constraints are partially inconsistent, $0<$ Cons $<1$ and a relaxation of the flexible constraints is implicitly performed, the higher the Cons is, the less the soaking time can be relaxed.

\subsection{A computation based on $\alpha$-cuts}

A first approach for finding a schedule that maximizes Sat is to transform the fuzzy problem into a series of classical HSP. Remember that the $\alpha$-cut of a fuzzy set $F$ is the classical set $F_{\alpha}=\left\{v, \mu_{F}(v) \geqslant \alpha\right\}$. For any $\alpha, \beta$ such that $\alpha \geqslant \beta, \quad F_{\alpha} \subseteq F_{\beta}$. Moreover, it holds that $\mu_{F}(v)=\sup \left\{\alpha, v \in F_{\alpha}\right\}$. This means that the fuzzy sets can be reconstructed from their $\alpha$-cuts: as soon as there is a $F_{\alpha}$ such that $v \in F_{\alpha}$, it holds that $\mu_{F}(v) \geqslant \alpha$; reciprocally, if $v \notin F_{\alpha}$, then $\mu_{F}(v)<\alpha$.

Hence

$$
\begin{aligned}
& \min _{O_{i, j}} \mu_{S_{i, j}}\left(t_{i, j+1}-t_{i, j}-c_{i, j+1}\right)= \\
& \left\{\begin{array}{c}
\min _{O_{i, j}} \sup \left\{\alpha \text { such that } t_{i, j+1}-t_{i, j}-c_{i, j+1} \in\left(S_{i, j}\right)_{\alpha}\right\}, \\
\sup \left\{\alpha \operatorname{such} \text { that } \forall O_{i, j}, t_{i, j+1}-t_{i, j}-c_{i, j+1} \in\left(S_{i, j}\right)_{\alpha}\right\} .
\end{array}\right.
\end{aligned}
$$

Moreover, when $F$ is a fuzzy interval, $F_{\alpha}$ is a classical interval defined as follows:

$F_{\alpha}=[L(F, \alpha), U(F, \alpha)]$,

where $L(F, \alpha)=\inf \left\{v, \mu_{m}(v) \geqslant \alpha\right\}$ and $U(F, \alpha)=\sup \{v$, $\left.\mu_{M}(v) \geqslant \alpha\right\}$.

For instance, $\left(S_{i, j}\right)_{1}$ is the interval defined by $L \times$ $\left(S_{i, j}, 1\right)=m_{i, j}^{*}$ and $U\left(S_{i, j}, 1\right)=M_{i, j}^{*}$ : This allows one to write

$$
\begin{aligned}
& \min _{O_{i, j}} \mu_{S_{i, j}}\left(t_{i, j+1}-t_{i, j}-c_{i, j+1}\right)= \\
& \quad \sup \left\{\alpha \text { such that } \forall O_{i, j},\right. \\
& \left.\quad L\left(S_{i, j}, \alpha\right) \leqslant t_{i, j+1}-t_{i, j}-c_{i, j+1} \leqslant U\left(S_{i, j}, \alpha\right)\right\} .
\end{aligned}
$$

Consider a sequence $s$ satisfying the crisp constraints (1)-(4), (6b). As soon as there is an $\alpha$ such that $s$ satisfies the $\alpha$-cuts of the chemical constraints:

$L\left(S_{i, j}, \alpha\right) \leqslant t_{i, j+1}-t_{i, j}-c_{i, j+1} \leqslant U\left(S_{i, j}, \alpha\right)$

then it holds that $\operatorname{Sat}(A) \geqslant \alpha$. Reciprocally, if $s$ violates one of the constraints (1)-(4), $\left(5^{\prime}\right),(6 b)$, then $\operatorname{Sat}(A)<\alpha$. Hence the following sketch of an algorithm can be used to find an admissible sequence that maximizes Sat. The idea is to sequentially solve $\alpha$-cuts of the fuzzy HSP with temporal bounds, until a precision bound $\varepsilon$ is reached. 
The exploration of the cuts follows a principle of dichotomy.

$\gamma:=1$

$\beta:=0$.

While $(\gamma-\beta>\varepsilon)$ do

$\alpha:=(\gamma+\beta) / 2$

Update the $L\left(S_{i, j}, \alpha\right)$ and the $U\left(S_{i, j}, \alpha\right)$.

Search for a solution of the linear problem that involves

the logical constraints (1)

the Initialization constraints (2)

the hoist capacity constraints (3)

the capacity constraints of the tanks (4)

the $\alpha$-cut of the chemical constraints (5c) the temporal constraint (6b)

If such a solution $s$ exists

then memorize $s$ (it is the best current sequence)

$$
\begin{aligned}
\beta & :=\alpha \\
\text { else } \gamma & :=\alpha
\end{aligned}
$$

Example of run: As initialization values, we know that there is a solution between levels 0 and 1 . The resolution of the cut of level $1 / 2$ is the first run. If the cut of level $1 /$ 2 accepts a solution, then the lower bound $\beta$ is improved (we know that Cons $\geqslant 1 / 2$ ). The algorithm then searches for a solution at level $(1+1 / 2) / 2=3 / 4$. Let us suppose that the corresponding system of linear inequations is inconsistent, the upper bound $\gamma$ is improved (we know that Cons $<3 / 4)$. The problem of level $(3 / 4+1 / 2) / 2=5 / 8$ is then solved. Assume that it is consistent: $\beta$ is improved to $5 / 8$. With $\varepsilon=0.2$, we can now stop the search.

\subsection{A direct computation of an optimal solution}

The previous algorithm is based on several calls to a linear program representing the $\alpha$-cuts of the fuzzy HSP. It results in a nearly optimal solution. Its quality depends on the precision degree $\varepsilon$ which has to be set by the user.

On the other hand, when the shape of the fuzzy intervals $S_{i, j}$ is trapezoidal, an optimal sequence can be obtained through a direct computation. Following the work of Zimmermann (1976) on fuzzy linear programming, when $S$ is a trapezoidal fuzzy set defined by the 4uple $\left(m_{*}, m^{*}, M^{*}, M_{*}\right), \mu_{S}(x)$ is the optimal solution of the program:

maximize $v$ under the constraints

$0 \leqslant v \leqslant 1$,

$v \leqslant \frac{x-m_{*}}{m^{*}-m_{*}}$

$v \leqslant \frac{x-M_{*}}{M^{*}-M_{*}}$
Hence the solutions that maximize Sat as defined in (8) are the solutions of the following mixed integer linear program:

maximize Sat under

the logical constraints (1)

the initialization constraints (2)

the hoist capacity constraints (3)

the capacity constraints of the tanks (4)

the temporal constraint (6b)

the chemical specific constraint that defines the degree of satisfaction

$$
\begin{aligned}
& 0 \leqslant S a t \leqslant 1, \\
& S a t \leqslant \frac{t_{i, j+1}-t_{i, j}-c_{i, j+1}-m_{i, j *}}{m_{i, j}^{*}-m_{i, j *}}, \\
& S a t \leqslant \frac{t_{i, j+1}-t_{i, j}-c_{i, j+1}-M_{i, j *}}{M_{i, j}^{*}-M_{i, j *}} .
\end{aligned}
$$

The inconsistency of DHSP can be due to other causes than temporal bounds. For instance, when small breakdowns occur (on the hoist or on the tanks) a Local Problem is generated in which no new carrier is considered-but the state of the line has changed: carriers have remained in the same position longer than planned. This Local Problem may have no solution so that all the in-process carriers can get off the line with respect to the ideal chemical constraints. In such a case, considering the makespan has no interest and the problem again consists in finding a schedule that maximizes the Local Problem satisfaction degree, Sat. The optimization of Sat in this case is performed so as to look for a minimal relaxation of the flexible constraints that enables consistency to be recovered. In practice, any of the previous methods can solve the problem.

Finally, a due date can be assigned to a new carrier, for instance because of an emergency. Likewise the resulting Local Problem can be inconsistent. In such a situation, the user may either maximize the satisfaction degree while satisfying the carrier due date, or adopt a true bi-criteria approach that will be developed in the next section.

\section{Minimization of the makespan in fuzzy HSP}

\subsection{Fuzzy HSP: a bi-criteria decision problem}

The first interest of a fuzzy HSP model appears when a local problem turns out to be inconsistent. A second point results from the management of the trade-off between quality and makespan. Indeed, when the problem is consistent according to the extreme chemical constraints, the user has two indices to evaluate the relevance of an admissible sequence: the satisfaction 
degree that estimates its quality or preference and the makespan. A sequence chosen because it minimizes the makespan can be a very bad choice in terms of quality, although there could be another sequence that would highly enhance this quality with a very slight modification of the makespan. Conversely, if the quality is chosen as the predominant criterion, we can obtain a very bad makespan although a slight relaxation of some soaking times in the limit expressed by their flexibility could lead to a better makespan. We have to face a bicriteria decision problem.

In a lot of industrial applications, the user rather looks for a trade-off between quality and throughput of the line. A possible solution would be to aggregate the two criteria, but according to which formula? As soon as the satisfaction degree and the makespan cannot be expressed on the same scale (e.g. as a cost), such a formula cannot be established. Rather than aggregating of the two evaluations under the form of a single criterion, we propose to leave the decision to the user, preferring a decision-support approach rather than an automated solution to the problem.

Presenting all sequences to the user is obviously uninteresting. A better solution consists in providing him with the terms of the trade-off: either compute what is the shortest makespan that reachable for each level of satisfaction (see Fig. 7); or determine the highest satisfaction degree reachable for each bound of the completion time ${ }^{3}$ (see Fig. 8). In other terms, only points which are pareto-optimal will be presented to the user.

Computing all the points in the previous curves may be very time consuming. This can indeed be equivalent to listing all admissible sequence and evaluating them in terms of satisfaction degree and makespan. We will thus only compute the more relevant points, namely:

(i) The point that corresponds to the best makespan that can be reached without any relaxation of the quality (with a satisfaction degree of 1).

(ii) Symmetrically, the point that corresponds to the highest degree of satisfaction for the shortest admissible makespan.

These two points are particularly relevant because they give a first approximation of the range of the tradeoff. Eventually, some intermediate points can be computed between these two bounds, for instance according to a precision level determined by the user. This precision level may pertain either to the satisfaction level (Fig. 9) or to the makespan (see Fig. 10). The user can then himself determine how to balance the two criteria, by selecting one pair (makespan, satisfaction degree) in the curve, i.e. an admissible sequence.

\footnotetext{
${ }^{3}$ In terms of fuzzy sets, this corresponds to determining the fuzzy set of optimal makespans.
}

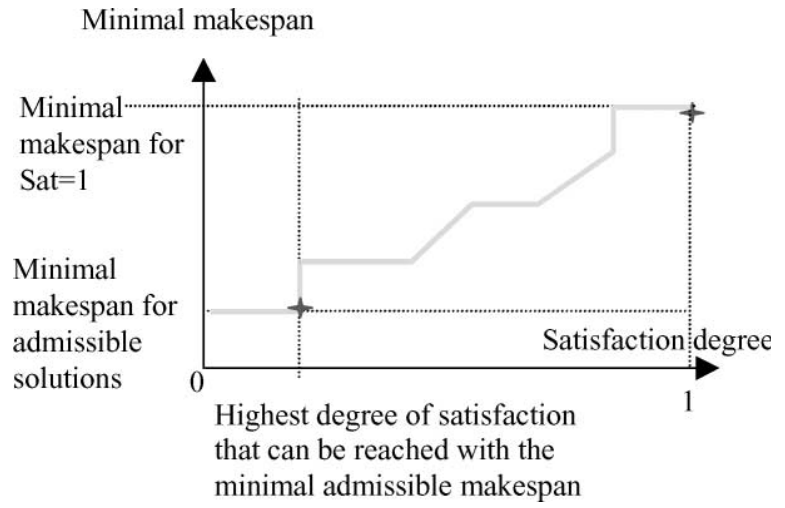

Fig. 7. Shortest makespan that can be reached for each level of satisfaction.

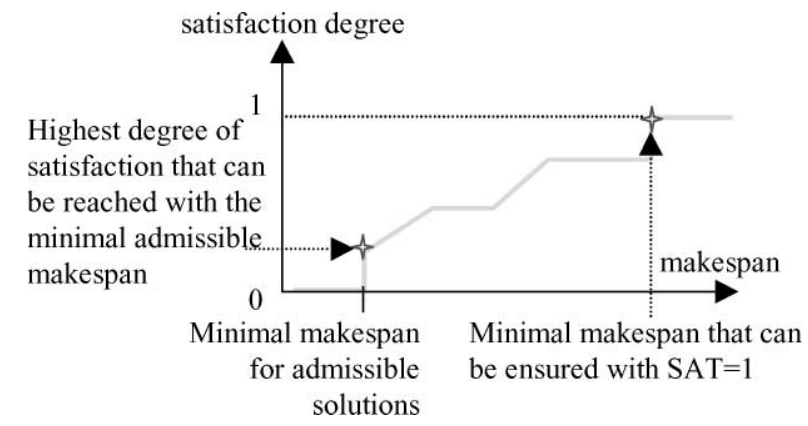

Fig. 8. Highest satisfaction degree that can be reached for each completion time.

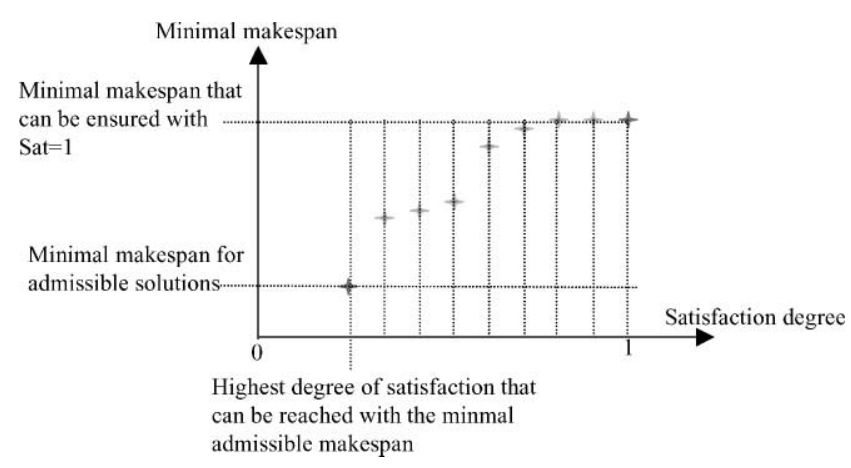

Fig. 9. Shortest makespan that can be reached for each level of satisfaction (discretized).

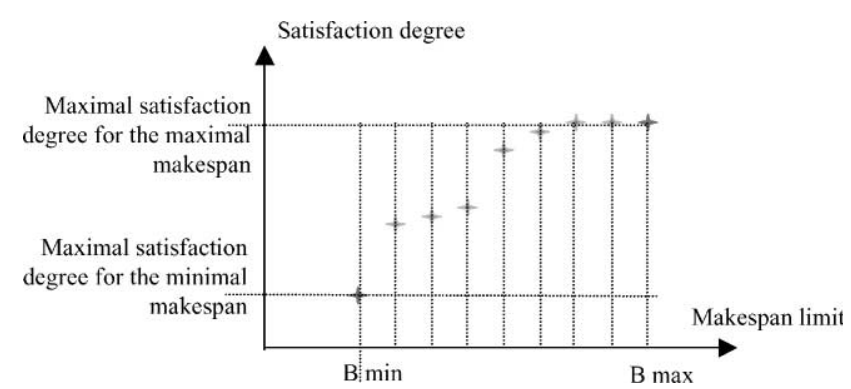

Fig. 10. Highest satisfaction degree that can be reached for each level of the makespan limit (discretized). 


\subsection{Computation of the shortest makespan for a quality degree of 1}

This first boundary point corresponds to the case where the chemical constraints are required to be satisfied at their maximal level, i.e. where quality degree of 1 is required. The soaking time intervals are thus reduced to their cuts of level 1 . The makespan is then used to discriminate among the different admissible sequences. We thus come back to a classical HSP local problem, which in turn comes down to the following linear program:

minimize $\max _{i} t_{i, n b_{-} o p(i)-1}$ under:

the logical constraints (1)

the initialization constraints (2)

the hoist capacity constraints (3)

the capacity constraints of the tanks (4)

the optimal chemical constraints:

$$
m_{i, j} * \leqslant t_{i, j+1}-t_{i, j}-c_{i, j+1} \leqslant M_{i, j}^{*}
$$

Again, this point is the best sequence that may be obtained if the user has modelled the fuzzy intervals so that the quality satisfaction degree is sufficient only at level 1 (no preferences), i.e. if quality was compulsory. However, this point is generally not satisfactory since this restrictive model does not take advantage of the flexibility of the chemical constraints to enhance the makespan.

\subsection{Computation of the maximal quality reachable for the shortest admissible makespan}

For this second boundary point, we are looking for the maximal satisfaction degree that can be reached when the makespan is at its minimal admissible value. This extreme point is computed in two stages.

Stage 1: we look for the value of the minimum admissible makespan. We thus come back to a classical HSP local problem. It comes down to the following linear program:

minimize $\max _{i} t_{i, n b_{-} o p(i)-1}$ under:

the logical constraints (1)

the initialization constraints (2)

the hoist capacity constraints (3)

the capacity constraints of the tanks (4)

the chemical limit constraints:

$$
m_{i, j} * \leqslant t_{i, j+1}-t_{i, j}-c_{i, j+1} \leqslant M_{i, j}^{*}
$$

Stage 2: Sat is used to discriminate among the admissible sequences that led to this makespan, i.e. to break ties.

Let $B$ denote the minimal makespan obtained at stage 1. The satisfaction of this optimal temporal bound is then ensured by the definition of the following constraint on the completion time:

$t_{i, n b_{-} o p(i)-1} \leqslant B$.

We thus obtain a second HSP, namely a flexible HSP with a temporal bound: it involves the crisp constraints (1)-(4), a temporal constraint of the form (6b) and the flexible chemical constraints (5). It can be solved according to the maximization of Sat using any of the methods described in Section 4.

\subsection{Computation of the shortest makespan with respect to a sufficient quality}

The computation of the Pareto-optimal points defining the minimal makespan reachable as a function of the satisfaction degrees can rely on $\alpha$-cuts in the flexible problem: for each value $\alpha$ of degree of satisfaction, $\alpha$ cuts of all the soaking time domains $S_{i, j}$ are computed. This results in a sequence of classical HSP:

Compute the minimal admissible makespan and its satisfaction degree $\beta$

For $\alpha:==\beta$ to 1 step $\varepsilon$

Compute $L\left(S_{i, j}, \alpha\right)$ and the $U\left(S_{i, j}, \alpha\right)$.

minimize $\max _{i} t_{i, n b_{-} o p(i)-1}$ under:

the logical constraints (1)

the initialization constraints (2)

the hoist capacity constraints (3)

the capacity constraints of the tanks (4)

the $\alpha$-cut of the chemical constraints (5b).

This computation based on $\alpha$-cuts can be applied for any shape of the fuzzy domains, i.e. not necessarily trapezoidal.

\subsection{Computation of the maximal quality reachable with respect to a sufficient completion time}

The computation of the Pareto-optimal curve defining the optimal satisfaction degree as a function of the completion time (see Fig. 10) rather comes down to a series of HSP problems with temporal bounds. As a consequence, this computation should be limited to trapezoidal fuzzy models of the soaking times.

The following sketch or algorithm can be used for instance:

Compute $B_{\max }$, the minimal makespan with a satisfaction degree of 1

Compute $B_{\min }$ the minimal admissible makespan and its satisfaction degree $\beta$

For $B:=B_{\min }$ to $B_{\max }$ step $\varepsilon$

maximize $S a t$ under

the logical constraints (1)

the initialization constraints (2)

the hoist capacity constraints (3)

the capacity constraints of the tanks (4) 
the temporal constraints: $\forall i, t_{i, n b_{-} o p(i)-1} \leqslant B$ the specific chemical constraints:

$$
\begin{aligned}
& 0 \leqslant \text { Sat } \leqslant 1 . \\
& \text { Sat } \leqslant \frac{t_{i, j+1}-t_{i, j}-c_{i, j+1}-m_{i, j *}}{m_{i, j}^{*}-m_{i, j *}}, \\
& \text { Sat } \leqslant \frac{t_{i, j+1}-t_{i, j}-c_{i, j+1}-M_{i, j *}}{M_{i, j}^{*}-M_{i, j *}} .
\end{aligned}
$$

The computation of a Pareto-optimal curve appears to be inappropriate in terms of computation time for an on-line use: too many sequences must be computed for a quick decision support. However, it is meaningful if the user looks for an evaluation of the trade-off between quality and makespan. It enables such questions to be answered as "which soaking time domains have to be selected in order to reach a desired throughput rate and what would be the consequences on quality". As a consequence, the curve of Pareto-optimal sequences is a tool for a line designer.

\subsection{Quick bi-criteria trade-off}

A more efficient variant of the latter approach can be proposed in the spirit of the STEM approach of interactive multi-criteria decision making (Benayoun et al., 1971). It consists of an iterative and interactive computation of the points that are the most interesting for the user (see Fig. 11).

The idea is first to determine the optimal makespan given a minimal satisfaction degree $\alpha$. This means solving the problem involving constraints (1)-(4) plus the chemical constraint (5c) reduced to an $\alpha$-cut of the interval $S_{i, j}$.

Then, the optimal makespan is known and the user can himself determine to what extent he accepts to decrease this shortest makespan so as to increase the degree of quality. In other words, the quality degree Sat is then used to discriminate from the admissible sequences the one that leads to this makespan with $a$ given tolerance $\varepsilon$. From a practical point of view, the

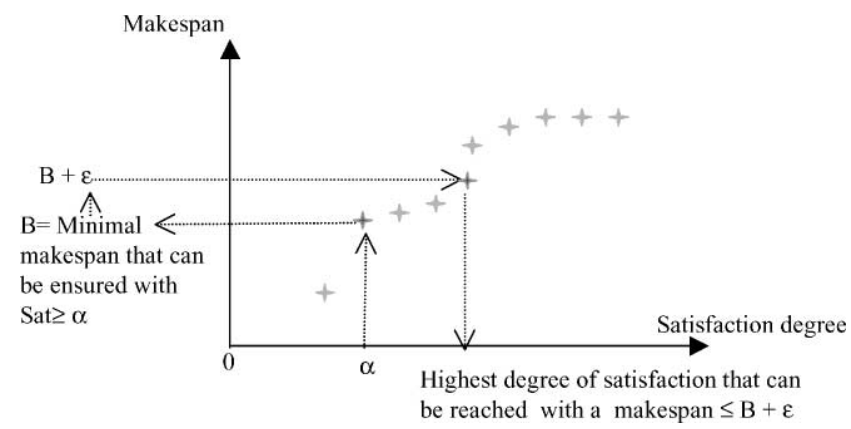

Fig. 11. A quick trade-off between makespan and satisfaction degree (the points in light grey are not computed in practice. following constraint on the completion time is set:

$t_{i, n b_{-} o p(i)-1} \leqslant B+\varepsilon$,

and we obtain a flexible HSP with temporal bounds that involves the crisp constraints (1)-(4), a temporal constraint of the form (6b) and the flexible chemical constraints (5). It can be solved maximixing Sat using any of the methods described in Section 4. Notice that the entire procedure reaches a trade-off between makespan and quality in only two invocations of a one-criterion-optimization routine.

\section{Conclusions}

The HSP problem has been studied considering that processing times on workstations can be chosen within fuzzy intervals. In practice, the degree of satisfaction of a fuzzy interval refers to a quality evaluation of the chemical treatment. Since a reaction of bad quality in a tank cannot be counterbalanced by a better reaction in another tank, the satisfaction degree of a carrier is evaluated as the lowest degree of satisfaction of its soaking operations. Likewise, we choose to evaluate the satisfaction degree of the entire fuzzy HSP as the lowest satisfaction degree of its carriers. ${ }^{4}$ It should be noticed that this approach allows the handling of partially conflicting constraints. Indeed, when temporal bounds are required, it may happen that no sequence can perfectly satisfy all the constraints. An implicit relaxation of the flexible constraints on the soaking time is performed: the higher the quality degree is, the less the ideal soaking times are relaxed.

From a practical point of view, the computing tools that we have proposed rely on a representation of the problem under the form of a linear program. The interest of this formulation is its generality, but it does not take advantage of the particularities of the problems, e.g. of the heuristics that are specific to HCSP. A more dedicated algorithm of fuzzy HSP is still to be developed. Now, the question is: is the computational cost of handling of flexibility reasonable compared with the cost of classical HSP? From a theoretical point of view, the answer is obviously affirmative, since we have shown that a fuzzy HSP is equivalent to a sequence of classical HSP: the order of magnitude of the theoretical complexity does not change. As it has already been seen when introducing soft constraints into scheduling (see e.g. Fargier, 1996), the information about the flexibility ranges in duration can in practice enhance the search for solutions since it can help the

\footnotetext{
${ }^{4}$ More complex aggregations of a carrier might have been considered such as the number of carriers whose satisfaction degree is less than a fixed number, a lexicographical classification of satisfaction degrees,... They would have resulted in longer computation time but would not have jeopardized our approach.
} 
software set the operations in the center of their windows.

The generic DHSP models that we have proposed thus lead to a trade-off between two criteria: quality and the line throughput. Rather than an aggregation of the two evaluations under the form of a single criterion, we have proposed to leave the decision to the user, preferring a decision-support approach. We have presented different solving processes showing their relevance in different decision support situations. The last approach consists of an interactive determination of the attitude of the decision maker in front of the bicriteria decision problem: a good trade-off between quality satisfaction and makespan can be reached quickly.

\section{References}

Armstrong, R., Lei, L., Shanhong, G., 1994. A bounding scheme for deriving the minimal cycle time of a single-transporter $N$-stage process with time-window constraints. European Journal of Operational Research 75, 1-11.

Benayoun, R., de Montgolfier, J., Tergny, J., Laricev, O., 1971. Linear programming with multiple objective function: the STEP method (STEM). Mathematical Programming 1, 366-375.

Bloch, C., Manier, M.A., 1999. Notation and typology for the hoist scheduling problem. Proceedings of the IEEE SMC Conference (SMC'99), Vol. 4, Tokyo, Japan, 12-15 October, pp. 475-480.

Bloch, C., Varnier, C., Baptiste, P., 1996. Applying stochastic methods to the real time hoist scheduling problem. Proceedings of the Computational Engineering in Systems Application Multiconference (CESA'96)-Symposium on Discrete Events and Manufacturing Systems, Lille, France, 9-12 July, pp. 479-484.

Caux, C., Fleury, G., Gourgand, M., Kellert, P., 1992. Couplage simulation à évènements discrets-méthodes stochastiques pour l'ordonnancement d'un atelier de traitement de surface. Proceedings of the European Simulation Multiconference (ESM'92), York, UK, 1-3 June, 1992.

Caux, C., Fleury, G., Gourgand, M., Kellert, P., 1995. Couplage méthodes dordonnancement-simulation pour lordonnancement de systèmes industriels de traitement de surface. RAIRO 29 (4), 391.

Chanas, S., Kamburowski, J., 1981. The use of fuzzy variables in PERT. Fuzzy Sets and Systems 5, 11-19.

Chen, H., Chu, C., Proth, J.M., 1998. Cyclic scheduling of a hoist with time window constraints. IEEE Transactions on Robotics and Automation 14 (1), 144-152.

Dubois, D., 1989. Fuzzy knowledge in an artificial intelligence system for job-shop scheduling. In: Evans, G., Karwowski, W., Wilhelm, M. (Eds.), Applications of the Fuzzy Set Methodologies in Industrial Engineering. Elsevier, New York, pp. 73-89.

Dubois, D., Fargier, H., Prade, H., 1995. Fuzzy constraints in jobshop scheduling. Intelligent Manufacturing 6, 215-234.

Dubois, D., Prade, H. (with the collaboration of Farreny H. MartinClouaire R. Testemale C.) 1988. Possibility Theory-An Approach to Computerized Processing of Uncertainty. Plenum Press, New York.

Fargier, H., 1996. Fuzzy scheduling: principles and experiments. In: Dubois, D., Prade, H., Yager, R.R. (Eds.), Fuzzy Information Engineering: A Guided Tour of Applications. Wiley, New York, pp. 655-669.

Fargier, H., Thierry, C., 1999. The use of qualitative decision theory in manufacturing planning and control: recent results in fuzzy master production scheduling. In: Slowinski, R., Hapke, M. (Eds.), Advances in Scheduling and Sequencing under Fuzziness, Studies in Fuzziness and Soft Computing Series. Springer, Berlin, pp. $45-59$.

Fleury, G., Goujon, J.Y., Gourgand, M., Lacomme, P., 1996. Hoist Scheduling Problem containing a fixed number of carriers, solved with an opportunistic approach. Proceedings of the Computational Engineering in Systems Application Multiconference (CESA'96)Symposium on Discrete Events and Manufacturing Systems, Lille, France, July, pp. 473-478.

Fortemps, P., 1997. Jobshop scheduling with imprecise durations: a fuzzy approach. IEEE Transactions on Fuzzy Systems 5 (4), 557-569.

Ge, Y., Yih, Y., 1995. Crane scheduling with time windows in circuit board production lines. International Journal of Production Research 33 (5), 1187-1189.

Hanen, C., 1994. Periodic scheduling of several hoists. Proceedings of the fourth International Workshop on Project Management and Scheduling, Leuven, Belgium, 12-15 July, pp. 108-110.

Hapke, M., Jaszkiewicz, A., Slowinski, R., 1994. Fuzzy project scheduling system for software development. Fuzzy Sets and Systems 67 (1), 101-117.

Hapke, M., Slowinski, R., 1996. Fuzzy priority heuristics for project scheduling. Fuzzy Sets and Systems 83, 291-299.

Ishibushi, H., Yamamoto, N., Misaki, S., Tanaka, H., 1994a. Local search algorithms for flow-shop scheduling with fuzzy due-dates. International Journal of Production Economics 33, 53-66.

Ishibushi, H., Yamamoto, N., Misaki, S., Tanaka, H., 1994b. Genetic algortihms and neighborhood search algorithms for fuzzy flowshop scheduling problems. Fuzzy Sets and Systems 67 (1), 81-100.

Ishii, H., Tada, M., Masuda, T., 1992. Two scheduling problems with fuzzy due-dates. Fuzzy Sets and Systems 46, 339-347.

Kerr, R.M., Walker, R.N., 1989. A job shop scheduling system based on fuzzy arithmetics. Proceedings of the Third International Conference on Expert Systems and the Leading Edge in Production and Operation Management, Hilton Head, SC, pp. 433-450.

Lacoste, M.A., Baptiste, P., 1992. Constraint logic programming approach for the $N$-periodic hoist scheduling problem. Proceedings of the Third International Workshop on Project Management on Scheduling, Como, Italy, 6-8 July.

Lamothe, J., Correge, M., Delmas, J., 1995. Dynamic heuristic for the real time hoist scheduling problem. Proceedings of ETFA'95, Symposium on Emerging Technologies and Factory Automation (INRIA/IEEE), Paris, France, 10-13 October, Vol. 2, pp. 161-168.

Lamothe, J., Thierry, C., Delmas, J., 1996. A multihoist model for the real time hoist scheduling problem. Proceedings of the Computational Engineering in Systems Application Multiconference (CESA'96)-Symposium on Discrete Events and Manufacturing Systems, Lille, France, 9-12 July, pp. 461-466.

Lei, L., Wang, T.J., 1994. Determining the optimal cyclic hoist schedules in a single-hoist electroplating line. IIE Transactions 2, 25-33.

Lim, J.M., 1997. A genetic algorithm for a single hoist scheduling in the printed-circuit-board electroplating line. Computers Industrial Engineering 33 (3-4), 789-792.

Lootsma, F.A., 1989. Stochastic and fuzzy PERT. Journal of Operational Research 43, 174-183 (in European).

Min, H.-S., Yih, Y., Kim, C.-O., 1998. A competitive neural network approach to multi-objective FMS scheduling. International Journal of Production Research 36 (7), 1749-1765.

Nasution, S.H., 1993. Fuzzy critical path method. IEEE Transactions on Systems, Man and Cybernetics 24 (1), 48-57.

$\mathrm{Ng}$, W.C., 1996. A branch and bound algorithm for hoist scheduling of a circuit board production line. International Journal of Flexible Manufacturing Systems 8 (1), 45-65. 
Norre, S., 1999. Coupling heuristic and simulation for the hoist scheduling problem multi-products multi-functions with empty carriers. APII-JESA 33 (4), 439-469.

Phillips, L.W., Unger, P.S., 1976. Mathematical programing solution of a Hoist Scheduling Program. AIIE Transactions 8 (2), 219-225.

Ptuskin, A.S., 1995. No-wait periodic scheduling of non-identical parts in flexible manufacturing with fuzzy processing times. In: Levner, E. (Ed.), Proceedings of the International Workshop on Intelligent Scheduling and Flexible Manufacturing Systems, CTEH, Hohn (Israel), 2 July 1995, pp. 210-222.

Shapiro, G.W., Nuttle, H.L.W., 1988. Hoist scheduling for a PCB electroplating facility. IIE Transactions 20 (2), 157-167.

Song, W., Zabinsky, Z.B., Storch, R.L., 1993. An algorithm for scheduling a chemical processing tank line. Production Planning and Control 4 (4), 323-332.
Sun, T.C., Lai, K.K., Lam, K., So, K.P., 1994. A study of heuristics for bidirectional multi-hoist production scheduling systems. International Journal of Production Economics 33, 207-214.

Yih, Y., 1990. Trace-driven knowledge acquisition (TDKA) for rulebased real time scheduling systems. Journal of Intelligent Manufacturing 1, 217-230.

Yih, Y., 1994. An algorithm for hoist scheduling problems. Inter national Journal of Production Research 32 (3), 501-516.

Yin, N.C., Yih, Y., 1992. Crane scheduling in a flexible electroplating line: a tolerance based approach. Journal of Electronics Manufacturing 2, 137-144.

Zimmermann, H.J., 1976. Description and optimization of fuzzy systems. International Journal of General Systems 2, 209-215. 